\title{
EFFECTS OF VOLUMETRIC BOUNDARY CONDITIONS ON THE COMPRESSIVE MECHANICS AND MODELING OF PASSIVE SKELETAL MUSCLE
}

\author{
Anurag J. Vaidya (1), Benjamin B. Wheatley (2)
}

\author{
(1) Biomedical Engineering \\ Bucknell University \\ Lewisburg, PA, USA
}

\author{
(2) Mechanical Engineering \\ Bucknell University \\ Lewisburg, PA, USA
}

\section{INTRODUCTION}

For over two decades, computational models of human bodysuch as the Toyota THUMS model- have been used in automobile safety [1]. These models rely on accurate material properties for each tissue. However, the compressive behavior of skeletal muscle is not fully understood, particularly regarding the differences in muscle response to in vivo loading conditions [2]. It is likely that in vivo muscle experiences a variation between confined and unconfined volumetric boundary conditions, but nearly all previous studies investigating passively compressed tissue have focused on muscle in unconfined compression (UC) $[2,3]$. One study has investigated muscle under anisotropic semi-confined compression [4], however none have studied muscle in fully confined compression (CC). Thus, we have investigated the effects of volumetric boundary conditions (UC and CC) on the stress relaxation of skeletal muscle. Moreover, a finite element model simultaneously characterizing muscle behavior in both boundary conditions is explored.

\section{METHODS}

Using an impermeable steel well, transverse oriented plugs $(\varnothing=$ $6.4 \mathrm{~mm}$ ) of porcine tibialis anterior (TA) from six animals were subject to CC stress relaxation tests. Two testing configurations were employed with $\mathrm{n}=8$ for each: fast-compression (strain rate $=15 \% / \mathrm{s} \epsilon=15 \%$ ) and slow-compression (strain rate $=1.5 \% / \mathrm{s}, \epsilon=1.5 \%$ ). Similarly, transverse oriented cubes (thickness $=7.9 \pm 0.5 \mathrm{~mm}$ ) of TA from six animals were exposed to stress relaxation tests under UC in two testing configurations with $\mathrm{n}=8$ for each: fast-compression (strain rate $=$ $40 \% / \mathrm{s}, \epsilon=40 \%$ ) and slow-compression (strain rate $=5 \% / \mathrm{s}, \epsilon=5 \%$ ). All samples relaxed for 400 s after compression. Data was collected by a $10 \mathrm{~N}$ Instron load cell at $100 \mathrm{~Hz}$. Testing was completed within eight hours of sacrifice to reduce the effects of rigor mortis. Muscle samples were hydrated by physiological buffer solution. First Piola-Kirchoff stress and nominal strain were determined through post-hoc analysis of initial specimen dimensions.

Three-dimensional finite element models of $\mathrm{UC}$ and $\mathrm{CC}$ geometries were developed in Abaqus (Dassault Systèmes). The UC geometry was reduced to a quarter of the sample by symmetry with 175 first-order 8-node hexahedral elements (type C3D8RH). This model was compressed by rigid platen and reaction force on platen was divided by initial area to acquire first Piola-Kirchhoff stress. The CC geometry was reduced to a two-dimensional axisymmetric model of cylinder with 96 first-order 4-node quadrilateral elements (type CAX4RH). Displacement was prescribed for top surface and as no lateral expansion occurred, first Piola-Kirchhoff stress was determined directly from model output. Displacements in both fast compression models simulated the slight experimental overshoot applied by the Instron.

A quasi-linear hyper-viscoelastic material formulation was chosen to model the behavior of skeletal muscle subject to both CC and UC. The model utilized third degree Yeoh form [5] of a polynomial hyperelastic strain energy density function $(\Psi(C)$ ) (Equation 1).

$$
\Psi(\mathbf{C})=\sum_{i=1}^{3} C_{i 0}\left(\bar{I}_{1}-3\right)^{i}+\sum_{i=1}^{3} \frac{1}{D_{i}}(J-1)^{2 i}
$$

Here $C_{i 0}$ and $D_{i}$ are material parameters, $\bar{I}_{1}$ is defined as $\bar{I}_{1}=\bar{\lambda}_{1}+$ $\bar{\lambda}_{2}+\bar{\lambda}_{3}$ where $\bar{\lambda}_{i}=J^{-\frac{1}{3}} \lambda_{i}\left(\lambda_{i}\right.$ are the principle stretches), and $J$ is the volume ratio. A Prony series viscoelastic model (Equation 2) was applied to the shear $\left(\bar{I}_{1}\right)$ and volumetric $(J)$ responses in Equation 1.

$$
K(\tau)=K_{\infty}+\sum_{i=1}^{4} K_{i} e^{-\tau / \tau_{i}^{K}} \quad G(\tau)=G_{\infty}+\sum_{i=1}^{4} G_{i} e^{-\frac{\tau}{\tau_{i}^{G}}}
$$


Here $K(\tau)$ is the time dependent bulk modulus and $G(\tau)$ is the time dependent shear modulus. $K_{\infty}$ and $G_{\infty}$ model long-term bulk and shear modulus respectively. $\tau_{i}^{K}$ and $\tau_{i}^{G}$ are time constants $\left(\tau_{1}^{G}=\tau_{1}^{K}=0.05 s\right.$, $\tau_{2}^{G}=\tau_{2}^{K}=1 s, \tau_{3}^{G}=\tau_{3}^{K}=20 s, \tau_{4}^{G}=\tau_{4}^{K}=400 s$ ). A power law model $\left(y=A x^{-B}\right)$ was also fit to all relaxation data to characterize and compare relaxation behavior between testing groups.

The finite element model consisted of fourteen parameters (6 hyperelastic, 8 viscoelastic). Parameter identification was achieved by a nonlinear least-squares deterministic optimization (lsqnonlin in MATLAB). The error function (Equation 3) was minimized to simultaneously fit experimental UC and $\mathrm{CC}$ fast-compression data $\left(t_{\text {peak }}\right.$ is time of peak experimental stress). UC and CC slowcompression data was then predicted by this optimized model.

error $=\sum_{t=0}^{t_{\text {peak }}} t *\left(\sigma_{\text {model }}-\sigma_{\text {exp }}\right)+\sum_{t=t_{\text {peak }}}^{401} \frac{\left(\sigma_{\text {model }}-\sigma_{\text {exp }}\right)}{t}$

\section{RESULTS}
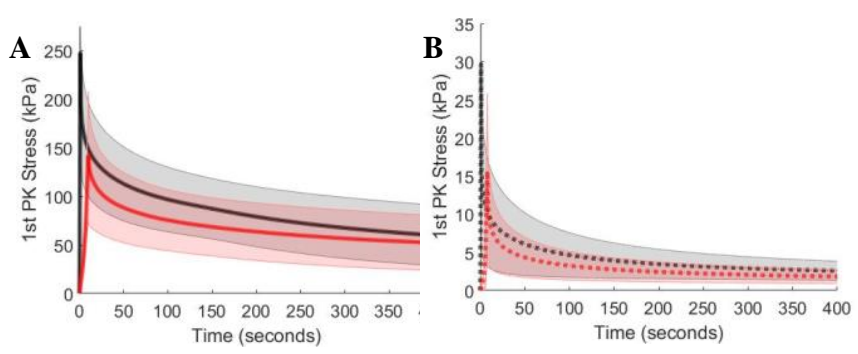

Figure 1: Average stress relaxation curves for (A) CC fast and slow compression (B) UC fast and slow compression with standard deviation

Figure 1 shows that despite lower strain levels, muscle was stiffer in CC than in UC (p-value $<0.001$ ). In both $\mathrm{UC}$ and $\mathrm{CC}$, muscle stiffness increased with strain rate $(\mathrm{p}=0.02$ for $\mathrm{CC}, \mathrm{p}=0.03$ for $\mathrm{UC}$ ), which is consistent with previous studies [2]. Fitting the power law model $\left(\bar{R}^{2}=0.96\right)$ shows that relaxation behavior differed with boundary condition at both strain rates $(\mathrm{p}<0.001$ for slow compression, $\mathrm{p}=0.042$ for fast compression). Relaxation was independent of strain rate in UC $(\mathrm{p}=0.47)$, whereas it depended on strain rate in $\mathrm{CC}(\mathrm{p}=0.030)$ (Table 1). The FEA model provided excellent simultaneous fits to $\mathrm{CC}$ and UC fastcompression data (Figure 2 A-B) (Table 2). Optimized parameters (Table 3) were used to calculate initial shear modulus $(0.044 \mathrm{kPa})$ and initial bulk modulus (42.6 kPa). Approximately two thirds of muscle instantaneous shear and bulk moduli relaxed with the first time constant $(0.05 \mathrm{~s})$ for both CC and UC (Table 3$)$. The model showed acceptable validation for UC and CC slow-compression (Figure 2C-D, NRMSE UC $10.65 \%$, CC NRMSE 9.02\%).

Table 1: Power law B-parameter values \pm SD for different groups

\begin{tabular}{|c|c|c|c|c|}
\hline & CC Fast & CC Slow & UC Fast & UC Slow \\
\hline B value & $0.23 \pm 0.06$ & $0.15 \pm 0.07$ & $0.32 \pm 0.08$ & $0.35 \pm 0.04$ \\
\hline
\end{tabular}

Table 2: Error values for model-experiment comparisons

\begin{tabular}{|c|c|c|}
\hline Error Type & UC & CC \\
\hline Peak error & $0.05 \%$ & $4.72 \%$ \\
\hline NRMSE & $1.71 \%$ & $0.85 \%$ \\
\hline
\end{tabular}

Table 3: Optimized finite element model parameters

\begin{tabular}{|c|c|c|}
\hline Parameter Type & Parameter & Optimized value \\
\hline Hyperelastic $(\mathrm{MPa})$ & $\mathrm{C}_{10}, \mathrm{C}_{20}, \mathrm{C}_{30}$ & $2.22 \mathrm{e}-5,1.32 \mathrm{e}-4,3.16 \mathrm{e}-5$ \\
\hline Hyperelastic $\left(\mathrm{MPa}^{-1}\right)$ & $\mathrm{D}_{10}, \mathrm{D}_{20}, \mathrm{D}_{30}$ & $46.99,1.29,10 \mathrm{e} 10$ \\
\hline Shear Coefficients & $\mathrm{G}_{1}, \mathrm{G}_{2}, \mathrm{G}_{3}, \mathrm{G}_{4}$ & $0.68,0.13,0.097,0.077$ \\
\hline Bulk Coefficients & $\mathrm{K}_{1}, \mathrm{~K}_{2}, \mathrm{~K}_{3}, \mathrm{~K}_{4}$ & $0.62,0.091,0.093,0.17$ \\
\hline
\end{tabular}
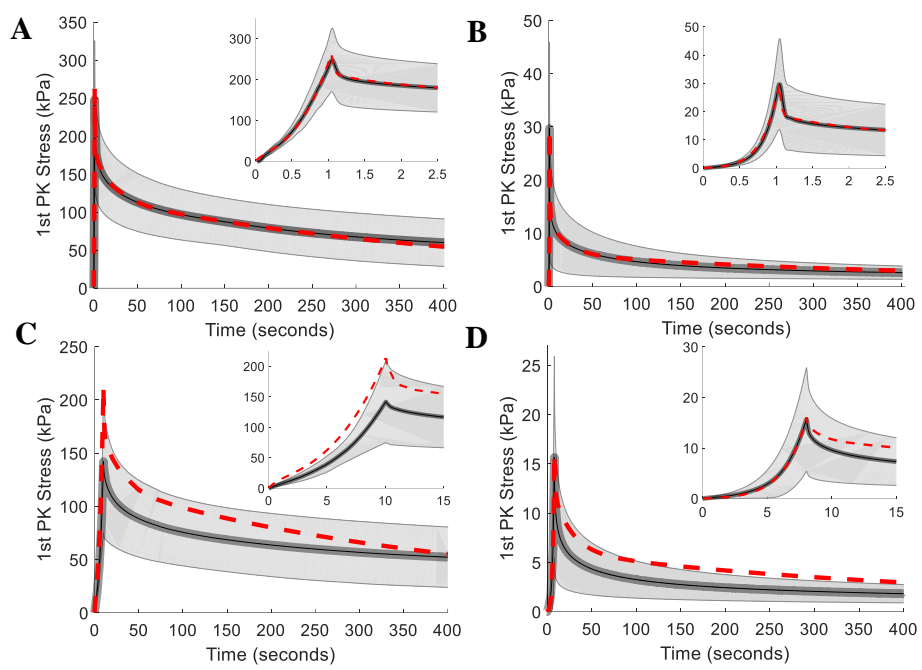

Figure 2: Average fast-compression experimental data (solid black) and optimized model (dashed red) with SD for (A) CC and (B) UC. Average slow-compression experimental data (solid black) and model predictions (dashed red) with SD for (C) CC and (D) UC.

\section{DISCUSSION}

This study presents new data of passively compressed muscle under different volumetric boundary conditions (UC versus CC) along with a quasi-linear hyper-viscoelastic finite element analysis. While a previous viscoelastic approach successfully modelled stress relaxation of muscle in UC [3], this study simultaneously fit stress relaxation data of muscle in CC and UC, thus providing greater accuracy for the volumetric behavior of skeletal muscle.

Since muscle's bulk modulus is three orders of magnitude larger than its shear modulus, muscle shows great resistance to volumetric deformations. This supports previous findings of near incompressibility of muscle $[2,4]$. The validation for CC slow compression suggests that there may be a mechanism in addition to viscoelasticity that dictates muscle stiffness and stress relaxation under volumetric deformations. The impermeable walls of the CC testing well may trap intramuscular fluid within the muscle. This boundary condition - along with previous studies showing non-negligible permeability of skeletal muscle [6]suggests that pressurization of incompressible fluid may contribute to the stiffness of muscle in CC. Such a model may induce non-linearity in the relaxation behavior of muscle in compression.

It is not yet entirely clear how differences in strain level and rate attribute to data presented in Figure 1. Studies at higher strain levels are required, nevertheless this study provides a robust model that effectively characterizes compressive behavior of skeletal muscles in different volumetric boundary conditions.

\section{ACKNOWLEDGEMENTS}

The authors thank Program for Undergraduate Research at Bucknell University for funding this study.

\section{REFERENCES}

[1] Toyota Motor Europe. "Toyota THUMS." Toyota Motor Europe

[2] Moh., M., J Mech Behav Biomed Mater 62 (2016): 468-480.

[3] Van Loocke, M., Journal of biomechanics 41.7 (2008): 1555-1566.

[4] Böl, M., J Mech Behav Biomed Mater 63 (2016): 115-124.

[5] Yeoh, H., Rubber Chemistry and technology 66.5 (1993): 754-771.

[6] Wheatley, B., Comput Meth Biomech Biomed Eng Imaging 20.6 (2017): 598-601. 
To SB3C Student Paper Competition Committee,

This letter serves as my attestation that Anurag Vaidya has completed the majority of the work (experimentation, analysis, and writing) presented in his SB3C BS level Student Paper Competition abstract. Anurag began working on the project December of 2017 and has been the sole student to partake in this research effort. Under my guidance, he completed all experimentation outlined in this work. Together, we developed two finite element models that Anurag used for the mentioned simulations. Anurag modified MATLAB code I developed during my $\mathrm{PhD}$ work to run the optimization. He completed all statistics and wrote the first draft of the abstract, both of which I reviewed and commented on as necessary.

Anurag is currently a sophomore in Biomedical Engineering at Bucknell University. His work on this project spans the spring of his first year, one summer as an undergraduate research assistant in my laboratory, and is continuing this now as a sophomore.

Thank you for this opportunity, and please do not hesitate to contact me with any questions.

Sincerely,
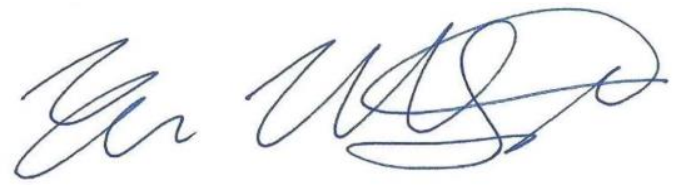

Benjamin Wheatley

Assistant Professor

570-557-3883 (office)

b.wheatley@bucknell.edu 